\title{
Digit ratio (2D:4D) and circulating testosterone, oestradiol, and progesterone levels across the menstrual cycle
}

\author{
Gareth Richards ${ }^{1}$, Magdalena Klimek ${ }^{2}$, Grazyna Jasienska², and Urszula M. Marcinkowska² \\ ${ }^{1}$ Autism Research Centre, Department of Psychiatry, University of Cambridge, UK \\ ${ }^{2}$ Department of Environmental Health, Faculty of Health Sciences, Jagiellonian University Medical College, \\ Poland \\ * Corresponding author address: Autism Research Centre, University of Cambridge, Douglas House, 18b \\ Trumpington Road, Cambridge, CB2 8AH; email: gvr22@cam.ac.uk
}

This is a pre-publication version of the following article:

Richards, G., Klimek, M., Jasienska, G., \& Marcinkowska, U. (2018). Digit ratio (2D:4D) and circulating testosterone, oestradiol, and progesterone levels across the menstrual cycle. Early Human Development, $117, \quad 68-73$. https://doi.org/10.1016/j.earlhumdev.2017.12.006

\begin{abstract}
Background: Digit ratio (2D:4D) is used by researchers as an indicator of prenatal sex hormone exposure. Two previous studies have examined associations between 2D:4D and circulating sex steroid concentrations across the menstrual cycle in adult females. One reported that digit ratio correlated positively with oestradiol levels, whereas the other found no such effect; neither observed significant associations with progesterone.
\end{abstract}

Aims: To examine associations between 2D:4D, as well as asymmetry (i.e. right minus left 2D:4D), and circulating sex steroids across the menstrual cycle.

Study design: Correlational.

Subjects: 32 naturally cycling adult females from rural southern Poland.

Outcome measures: Salivary oestradiol, progesterone, testosterone, and testosterone to oestradiol ratio $(\mathrm{T}: \mathrm{O})$ measured during the follicular, peri-ovulatory, and luteal phases. 
Average levels across the cycle were also examined.

Results and conclusions: Asymmetry in digit ratio correlated positively with oestradiol at each phase, as well as with average levels across the cycle. Each association, other than that relating to average levels, remained statistically significant after a range of covariates had been controlled for. No other significant correlations were observed between digit ratio variables and circulating hormone levels. Our results might suggest that low exposure to androgens and/or high exposure to oestrogens during gestation is a predictor of high oestradiol levels in naturally cycling females of reproductive age. However, considering that it was asymmetry in digit ratio, and not either right or left 2D:4D, that was a significant predictor, it is also possible that these effects reflect more general associations between bilateral asymmetry and circulating oestradiol levels.

\section{Introduction}

Manning et al. [1] reported significant correlations between circulating sex steroids and 2D:4D in a sample of 131 (69 men, 62 women) participants attending an infertility clinic. Oestrogen was positively related to $2 \mathrm{D}: 4 \mathrm{D}$, and the relationship was independent of sex, age, height and weight. Although testosterone was not measured in women, it was negatively related to $2 \mathrm{D}: 4 \mathrm{D}$ in men. All relationships were stronger for the right hand (R2D:4D) relative to the left (L2D:4D). Manning et al. [1] suggested that these correlations between 2D:4D and circulating sex steroids reflected echoes of causal associations between prenatal sex steroids and 2D:4D, and that R2D:4D was more sensitive to the effects of prenatal sex hormones. Later, the variable $\mathrm{D}_{[\mathrm{R}-\mathrm{L}]}(\mathrm{R} 2 \mathrm{D}: 4 \mathrm{D}-\mathrm{L} 2 \mathrm{D}: 4 \mathrm{D})$ was introduced to reflect this right-sided effect in the expression of 2D:4D (see Discussion of $\mathrm{D}_{[\mathrm{R}-\mathrm{L}]}$ by Manning [2], p. 21-22). Manning [2] (p. 37-38, Fig. 2.8) found that high $\mathrm{D}_{[\mathrm{R}-\mathrm{L}]}$ (i.e. relatively high L2D:4D compared to R2D:4D) was associated with high oestrogen levels in the same sample of men and women attending an infertility clinic reported on by Manning et al. [1], although the effect was no longer significant after controlling for sex.

The links between 2D:4D, prenatal testosterone and oestrogen and their receptors were confirmed in mice by Zheng \& Cohn [3] (see also Manning [4]). Mice show a similar sex difference in 2D:4D as humans (males < females), and in their development the sexual 
dimorphism of R2D:4D appears earlier than the sex difference in L2D:4D [3]. In humans, the effect size for the sex difference in R2D:4D has been reported to be larger than that of L2D:4D [5]. The side difference in sensitivity to sex steroids has also been shown in studies of 46XY individuals with a female phenotype (i.e. complete androgen insensitivity syndrome [CAIS], a condition characterised by non-functional or absent androgen receptors due to genetic mutations in the androgen receptor gene). Such individuals have high "female-type" digit ratios in comparison to male norms, and the effect size for this is larger for R2D:4D than L2D:4D $[6,7]$.

There is conflicting evidence for correlations between circulating sex steroids and 2D:4D. The original reports of links between circulating sex steroids and digit ratio variables $[1,2,8]$ considered an unusual population (i.e. men and women attending infertility clinics), and so the findings may not be generalisable. Regarding normative samples of men and women, some studies have observed significant correlations between 2D:4D and circulating levels of sex steroids [9,10], whereas others have not [8,11] (see Hönekopp et al. [11] for a review). However, the position regarding $\mathrm{D}_{[\mathrm{R}-\mathrm{L}]}$ and testosterone and oestrogen is different. Men who are subjected to challenge (e.g. physical exercise and/or aggressive encounters) show marked spikes in sex steroid levels. In such situations, $D_{[R-L]}$ has been reported to be a correlate of circulating levels of testosterone and oestrogen in men but not women [12,13] (see also, Manning et al. [14]).

In premenopausal women who have regular cycles, there may be correlations between $D_{[R-L]}$ and oestrogen levels across their cycle. McIntyre et al. [15] reported positive correlations between oestradiol and R2D:4D and $\mathrm{D}_{[\mathrm{R}-\mathrm{L}]}$ in a sample of normally cycling women. Klimek et al. [16] did not replicate this finding for R2D:4D in a larger sample (and did not examine $\mathrm{D}_{[\mathrm{R}-}$ L]). Thus, there remains the possibility that $D_{[R-L]}$ may be positively correlated with oestrogen across the cycle. The present study addresses this possibility and considers the relationship between $\mathrm{D}_{[\mathrm{R}-\mathrm{L}]}$ (and 2D:4D) and salivary oestradiol, progesterone, testosterone, and testosterone to oestradiol ratio (T:O) measured during the follicular, peri-ovulatory, and luteal phases of the cycle.

\section{Materials and methods}

\subsection{Participants}


Thirty-two females aged 22-37 $(M=30.3, S D=4.83)$ from the Mogielica Human Ecology Study Site in rural southern Poland [17] took part in the current research, none of whom had been pregnant, breastfeeding, or taking hormonal contraception for at least three months prior to the study. The majority $(30,93.8 \%)$ reported that they were in a relationship, and most (23, $71.9 \%$ ) had been pregnant before. The number of pregnancies reported ranged from 0 to 4 ( $M$ $=1.38, S D=1.1)$, and the women had between 0 and 3 children $(M=1.28, S D=0.958)$. Age of menarche ranged from 10 to $17(M=13.31, S D=1.6)$, and only $5(15.6 \%)$ reported experiencing differences in the length of consecutive cycles larger than \pm 5 days (range $=25$ 40 days, $M=29, S D=3.47$ ). The study was granted approval by the Jagiellonian University Bioethical Committee. All procedures were undertaken with the understanding and written consent of each subject, and in compliance with national legislation and the Code of Ethical Principles for Medical Research Involving Human Subjects of the World Medical Associations (Declaration of Helsinki).

\subsection{Hormone measurements}

Phases of the menstrual cycle were initially determined via self-reported ovulation strip tests, which were based on luteinizing hormone (LH) levels. These tests were performed from the 10th until the 20th days of the cycle or until a positive result was obtained. LH tests have been shown to be a highly accurate means of determining timing of ovulation [18]. Twenty-one (65.6\%) participants had a positive LH test, indicating that ovulation had been successfully detected, although it is noted that one participant did not have a strong positive result (i.e. lines visible were not intense red, but pale red). For the other 10 participants, occurrence of ovulation was suggested by $17-\beta$-oestradiol drop.

Per each participant, hormonal profiles of 17- $\beta$-oestradiol $(\mathrm{O})$, progesterone $(\mathrm{P})$ and testosterone $(\mathrm{T})$ were created based on 15 daily measurements centred around the ovulation for $\mathrm{O}$, the last 14 days of the cycle for $\mathrm{P}$, and 20 days centred around ovulation for T. Participants individually collected saliva at least 30 min after eating, drinking or smoking and froze all samples immediately after collection. Hormonal measurements were conducted using commercially available hormonal assays of DRG International Incl.: Elisa plates SLV4188 for 17- $\beta$-oestradiol (sensitivity: $0.4 \mathrm{pg} / \mathrm{ml}$, standard range: $1-100 \mathrm{pg} / \mathrm{ml}$ ), SLV3140 for $17-\alpha$ hydroxy-progesterone (sensitivity: $2.5 \mathrm{pg} / \mathrm{ml}$, standard range: $10-5000 \mathrm{pg} / \mathrm{ml}$ ) and SLV3013 for testosterone (sensitivity: $1.9 \mathrm{pg} / \mathrm{ml}$, standard range: $10-5000 \mathrm{pg} / \mathrm{ml}$ ). To ensure high quality of the measurements, all hormonal assays were conducted in duplicates. Quality of 
measurements was then controlled for each plate separately by including (in duplicates) samples of known concentrations ("pools") of O, P, and T (in total these control measurements consisted of 19 pools per plate). Inter- and intra-assay coefficients of variability (CVs) were computed: for oestradiol, inter-assay CV was $10.01 \%$ and intra-assay was $7.5 \%$; for progesterone, inter-assay $\mathrm{CV}$ was $14.1 \%$ and intra-assay was $4.9 \%$; for testosterone, inter-assay CV was $6.2 \%$ and intra-assay CV was $1.3 \%$ [19].

The hormonal measurements reported here for the follicular, peri-ovulatory, and luteal phases are those collected during the meetings held for each of the three phases (i.e. on the same days on which hand scans were made). Averaged hormonal levels were counted based on all daily measurements made: for $\mathrm{O}$ it was \pm 7 days around ovulation plus the ovulation day; for $\mathrm{P}$ it was the last 14 days of the cycle; for $\mathrm{T}$ it was \pm 10 days around ovulation.

\subsection{Digit ratio measurements}

Hand scans were made at three times during one menstrual cycle (one each phase of the cycle). The visits were scheduled for the early follicular phase (i.e. between day 2 and day 8 ), at the peri-ovulatory phase (not later than $72 \mathrm{~h}$ after a positive LH test, or, if no positive test result was obtained, on day 20), and in the mid-luteal phase (approximately one week post-ovulation). The second and fourth digits for each hand were measured from the hand scans separately by two researchers who were blinded to the identity of the participants. Each researcher made two sets of measurements, several weeks apart. Inter- and intra-observer reliability of measurement were examined using intraclass correlation coefficients (ICC) with two-way mixed-effects and absolute agreement definition. ICC for Observer 1 varied between 0.94 and 0.99; ICC for Observer 2 varied between 0.90 and 0.98 . The ICC between observers varied between 0.89 and 1.00 .

Klimek et al. [20] have already reported the R2D:4D, L2D:4D, and $\mathrm{D}_{[\mathrm{R}-\mathrm{L}]}$ values for this sample that were measured during the follicular, peri-ovulatory, and mid-luteal phases. As these values did not differ significantly between phases, average R2D:4D, L2D:4D, and $\mathrm{D}_{[\mathrm{R}-\mathrm{L}]}$ values were created from these separate sets of measurements (note that hand scans were not made during the follicular phase for three participants; in these cases, average digit ratio values were computed from the measurements taken during the peri-ovulatory and luteal phases only). This resulted in mean values of $0.977(S D=0.022)$ for $\mathrm{R} 2 \mathrm{D}: 4 \mathrm{D}, 0.97(S D=0.026)$ for $\mathrm{L} 2 \mathrm{D}: 4 \mathrm{D}$, and $0.007(S D=0.02)$ for $\mathrm{D}_{[\mathrm{R}-\mathrm{L}]}$. 


\subsection{Statistical procedures}

Of 16 hormone measures (i.e. follicular, peri-ovulatory, luteal, and average levels of oestradiol, progesterone, testosterone, and $\mathrm{T}: \mathrm{O}$ ), all but three (follicular oestradiol, peri-ovulatory testosterone, and average testosterone) were non-normally distributed, as index by ShapiroWilk test. To address this, and for ease of comparison, all hormone measures were transformed using natural logarithm (ln). R2D:4D, L2D:4D, and $\mathrm{D}_{[\mathrm{R}-\mathrm{L}]}$ were all normally distributed, and were not transformed. However, one outlier was identified for $D_{[R-L]}$. This was a particularly low (i.e. masculinised) value. On closer inspection, the participant to which this score belonged also recorded outlying (high) values for luteal progesterone, follicular oestradiol, luteal oestradiol, average oestradiol, and average testosterone. This participant was excluded from analyses in which $\mathrm{D}_{[\mathrm{R}-\mathrm{L}]}$ was used as a predictor of hormone levels.

Pearson's correlations were performed to determine whether digit ratio variables (i.e. R2D:4D, L2D:4D, and $\left.\mathrm{D}_{[\mathrm{R}-\mathrm{L}]}\right)$ were related to follicular, peri-ovulatory, luteal, and average levels of oestradiol, progesterone, testosterone, and T:O. These associations were then examined further with multiple linear regression, in which age, BMI, previous pregnancy (yes/no), age at menarche, and menstrual cycle length were entered as covariates. All statistical analyses were conducted using IBM SPSS version 24.

\section{Results}

Digit ratios for the right and left hands correlated positively, $r(30)=0.669, p<0.001$, and L2D:4D was lower than R2D:4D, although this latter effect narrowly missed being statistically significant, $t(31)=1.954, p=0.06$. Neither R2D:4D nor L2D:4D was significantly associated with any of the hormone variables, though there was a trend level $(p=0.067)$ positive correlation between L2D:4D and progesterone levels during the peri-ovulatory phase (for all correlations, see Table 1). $\mathrm{D}_{[\mathrm{R}-\mathrm{L}]}$ was positively correlated with oestradiol levels at each of the follicular, peri-ovulatory, and luteal phases, as well as with average levels across the menstrual cycle (see Fig. 1). There was also a trend level $(p=0.062)$ negative correlation between $D_{[R-L]}$ and $\mathrm{T}: \mathrm{O}$ measured at the follicular phase. However, although correlations were also in the negative direction for peri-ovulatory, luteal, and average $\mathrm{T}: \mathrm{O}$ levels, none approached statistical significance.

One participant had outlying (high) oestradiol scores at both the peri-ovulatory and luteal 
phases, and another participant had outlying (low) oestradiol levels at the luteal phase (after the hormone variables had been log transformed). For this reason, the significant associations between $\mathrm{D}_{[\mathrm{R}-\mathrm{L}]}$ and oestradiol at the peri-ovulatory and luteal phases were re-examined with the relevant participants removed from analysis. $\mathrm{D}_{[\mathrm{R}-\mathrm{L}]}$ remained significantly positively correlated with oestradiol at the peri-ovulatory, $r(26)=0.572, p=0.001$, and luteal phases, $r$ $(24)=0.409, p=0.038$, and so these participants were retained in the multivariate analyses presented below.

After statistically controlling for covariates (age, BMI, previous pregnancy [yes/no], age at menarche, and cycle length), $\mathrm{D}_{[\mathrm{R}-\mathrm{L}]}$ remained significantly positively correlated with oestradiol levels during the follicular, peri-ovulatory, and luteal phases, though the association with average oestradiol levels narrowly missed being statistically significant $(p=0.061)$. The marginally significant negative correlation between $\mathrm{D}_{[\mathrm{R}-\mathrm{L}]}$ and follicular $\mathrm{T}: \mathrm{O}$ that was observed at the univariate level was also observed here, though once again narrowly missed being statistically significant $(p=0.060)$. Likewise, a marginally significant positive correlation between L2D:4D and peri-ovulatory progesterone was observed again here ( $p=0.063$ ), along with a similar marginally significant effect for luteal progesterone $(p=0.059)$. No other effects of note were observed in this analysis (see Table 2). 
Table 1. Correlations between digit ratio variables and natural log transformed sex hormone levels across the menstrual cycle.

Note. All correlations are Pearson's (two-tailed); one participant was removed from analyses involving $D_{[R-L]}$ due to being an outlier; $P=$ progesterone, $O=$ oestradiol, $T=$ testosterone.

Bold $p$ values indicate statistical significance $(p<0.05)$.

\begin{tabular}{|c|c|c|c|c|c|c|c|c|c|c|}
\hline & & \multicolumn{3}{|c|}{ R2D:4D } & \multicolumn{3}{|c|}{ L2D:4D } & \multicolumn{3}{|c|}{$\mathbf{D}_{[\mathrm{R}-\mathrm{L}]}$} \\
\hline & & $n$ & $r$ & $p$ & $n$ & $r$ & $p$ & $n$ & $r$ & $p$ \\
\hline \multirow[t]{4}{*}{$\mathbf{P}$} & Follicular & 31 & -0.072 & 0.698 & 31 & 0.000 & 0.998 & 30 & -0.034 & 0.860 \\
\hline & Peri-ovulatory & 28 & 0.118 & 0.549 & 28 & 0.351 & 0.067 & 27 & -0.133 & 0.510 \\
\hline & Luteal & 28 & 0.137 & 0.487 & 28 & 0.232 & 0.235 & 27 & -0.019 & 0.924 \\
\hline & Average & 31 & 0.011 & 0.953 & 31 & 0.243 & 0.188 & 30 & -0.208 & 0.269 \\
\hline \multirow[t]{4}{*}{$\mathbf{O}$} & Follicular & 30 & 0.078 & 0.682 & 30 & -0.215 & 0.255 & 29 & 0.596 & 0.001 \\
\hline & Peri-ovulatory & 30 & 0.125 & 0.512 & 30 & 0.007 & 0.969 & 29 & 0.418 & 0.024 \\
\hline & Luteal & 29 & 0.148 & 0.443 & 29 & 0.057 & 0.768 & 28 & 0.434 & 0.021 \\
\hline & Average & 32 & 0.053 & 0.774 & 32 & -0.081 & 0.658 & 31 & 0.414 & 0.021 \\
\hline \multirow[t]{4}{*}{$\mathbf{T}$} & Follicular & 26 & -0.052 & 0.801 & 26 & -0.015 & 0.941 & 25 & 0.077 & 0.715 \\
\hline & Peri-ovulatory & 27 & 0.091 & 0.652 & 27 & 0.109 & 0.587 & 26 & 0.101 & 0.623 \\
\hline & Luteal & 27 & 0.147 & 0.464 & 27 & 0.292 & 0.139 & 26 & 0.030 & 0.885 \\
\hline & Average & 30 & 0.003 & 0.986 & 30 & 0.031 & 0.872 & 29 & 0.095 & 0.623 \\
\hline \multirow[t]{4}{*}{ T:O } & Follicular & 26 & -0.128 & 0.534 & 26 & 0.002 & 0.993 & 25 & -0.378 & 0.062 \\
\hline & Peri-ovulatory & 27 & -0.063 & 0.756 & 27 & 0.031 & 0.878 & 26 & -0.243 & 0.231 \\
\hline & Luteal & 27 & -0.050 & 0.803 & 27 & 0.102 & 0.614 & 26 & -0.296 & 0.142 \\
\hline & Average & 30 & -0.085 & 0.654 & 30 & 0.034 & 0.860 & 29 & -0.262 & 0.169 \\
\hline
\end{tabular}


Fig. 1. Pearson's correlations between $D_{[R-L]}$ and oestradiol measured at the follicular (a), peri-ovulatory (b), and luteal (c) phases, and averaged across the menstrual cycle (d).

Note. The correlations presented here include one participant who recorded outlying (high) values for oestradiol at both the peri-ovulatory and luteal phases, as well as another participant who recorded an outlying (low) value for oestradiol at the luteal phase; one participant was excluded from analysis due to having outlying (low) $D_{[R-L]}$ and outlying (high) follicular, luteal, and average oestradiol levels.
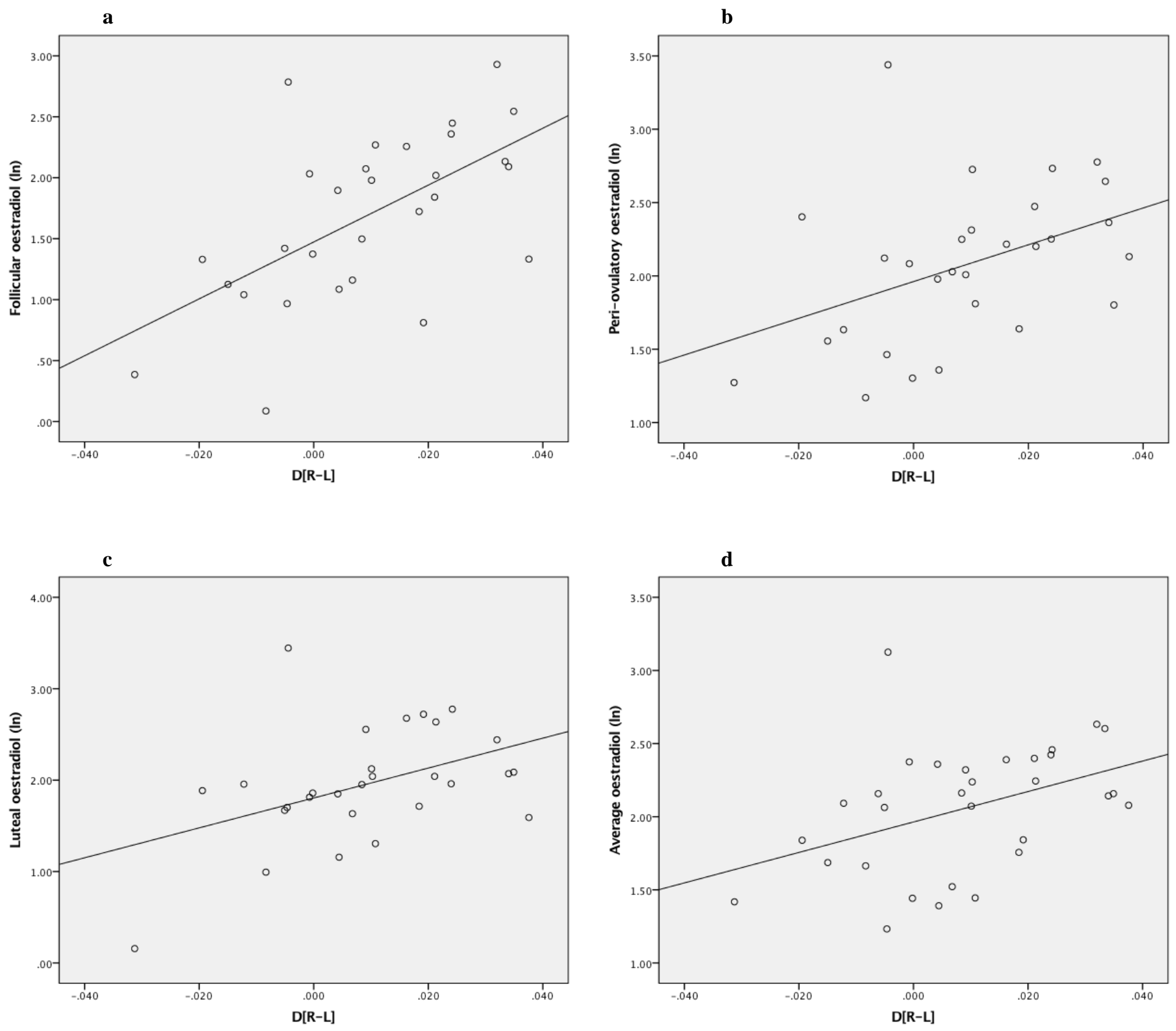
Table 2. Outcome of linear regression models, with digit ratio variable as predictor, hormone level (ln) as outcome, and age, BMI, cycle length, previous pregnancy (yes/no), and age or menarche as covariates. Note. One participant was removed from analyses involving $D_{[R-L]}$ due to being an outlier; $P=$ progesterone, $O=$ oestradiol, $T=$ testosterone. Bold $p$ values indicate statistical significance $(p<0.05)$.

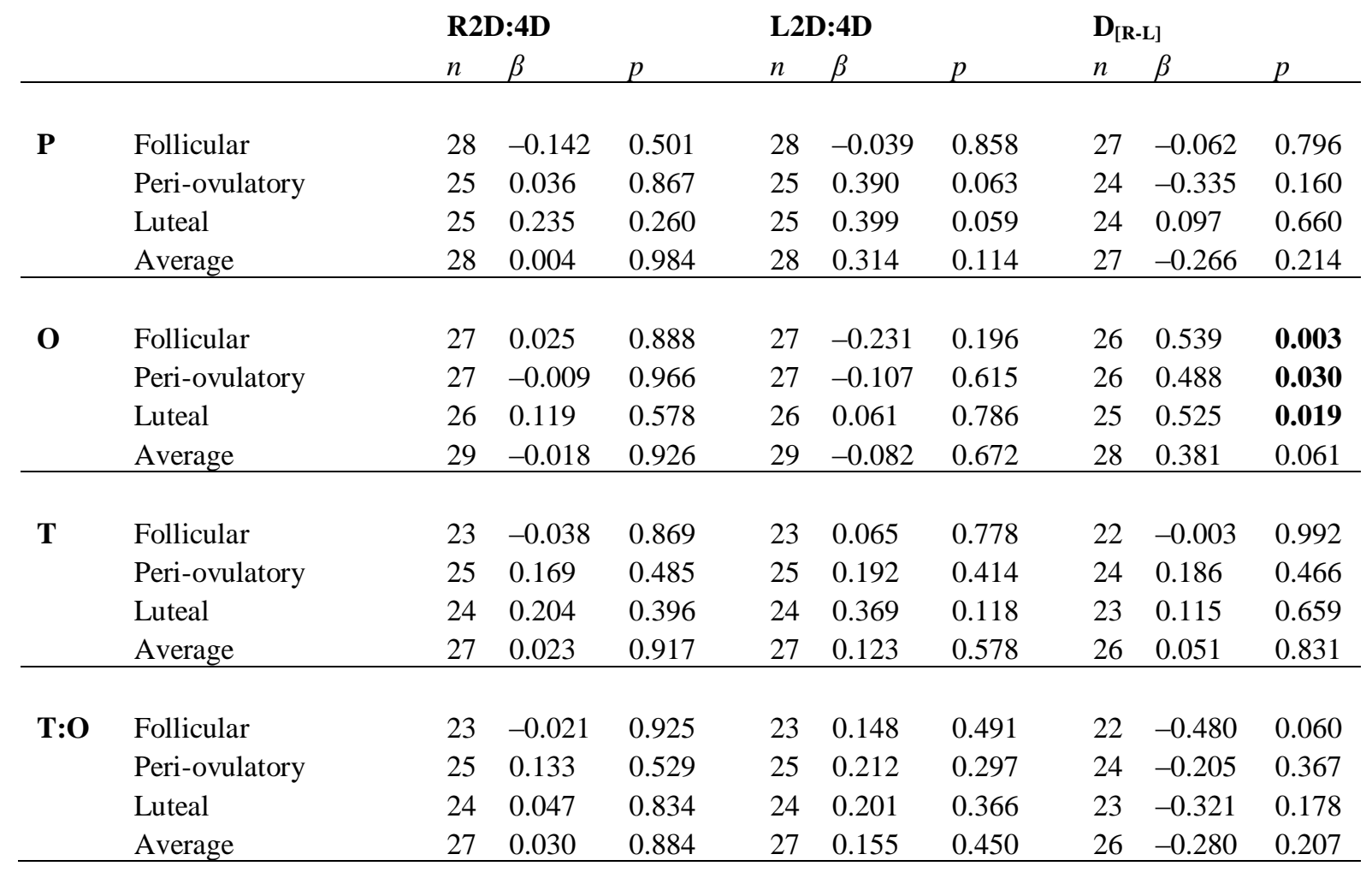

\section{Discussion}

The current study aimed to examine associations between digit ratio (2D:4D) and circulating sex hormones across a menstrual cycle in a sample of naturally cycling females. A previous study [15] reported positive correlations between digit ratio and oestradiol levels across the menstrual cycle, whereas a subsequent study [16] did not replicate these findings; neither observed significant associations between digit ratio and progesterone, and neither examined possible associations with testosterone or T:O. The current study observed significant positive correlations between directional asymmetry in digit ratio $\left(\mathrm{D}_{[\mathrm{R}-\mathrm{L}]}\right)$ and oestradiol, although no statistically significant effects were detected in relation to progesterone, testosterone, or T:O.

$\mathrm{D}_{[\mathrm{R}-\mathrm{L}]}$ was positively correlated with circulating concentrations of oestradiol during each menstrual phase examined, as well as with average levels across the cycle. The strength of the Pearson's correlations observed here $(r=0.414-0.596)$ was similar to that of the correlations 
reported by McIntyre et al. [15] ( $r=0.43-0.46)$. After controlling for age, BMI, previous pregnancy (yes/no), age at menarche, and menstrual cycle length, each effect remained statistically significant other than that relating to average oestradiol levels across the cycle. These findings are relevant in the context that $\mathrm{D}_{[\mathrm{R}-\mathrm{L}]}$ correlates negatively with the severity of premenstrual symptoms [21]. Although women with premenstrual syndromes typically have circulating oestrogen levels within the normal range [22], it could be that prenatal oestrogen (and/or prenatal testosterone) determines sensitivity to fluctuations in sex hormones during adult life. However, whereas McIntyre et al. [15] also observed significant positive correlations between R2D:4D and average oestradiol concentrations, the current study did not. Klimek et al. [16] also reported no significant associations between digit ratio and oestradiol levels (average, follicular, peri-ovulatory, or luteal) across a menstrual cycle in a larger sample $(n=$ 186) from a similar population as studied here. However, it is also noted that this study did not report $\mathrm{D}_{[\mathrm{R}-\mathrm{L}]}$ as a predictor variable.

It is unclear why the statistically significant associations between digit ratio and oestradiol observed in the current study all relate to $D_{[R-L]}$, yet some other studies have reported correlations between circulating sex hormone levels and R2D:4D and/or L2D:4D [9,15]. It is however noted that many studies of digit ratio and circulating sex hormones have not reported on $\mathrm{D}_{[\mathrm{R}-\mathrm{L}]}$ as a predictor variable $[8,9,10,16]$. One possibility is that $\mathrm{D}_{[\mathrm{R}-\mathrm{L}]}$ reflects differential expressions of sex hormone effects on the right and left sides of the body. This is compatible with Tanner's [23] suggestion that sexually dimorphic traits tend to be expressed in the "male form" more strongly on the right side of the body in men, and in the "female form" more strongly on the left side of the body in women. Further to this, Kimura [24] reported that males with a larger right than left testicle, and women with a larger right than left breast achieved higher scores on tests that favour males, and that the pattern was reversed for tests favouring females (see Manning [2], p. 21-22 for a discussion of these effects in relation to 2D:4D and $\left.\mathrm{D}_{[\mathrm{R}-\mathrm{L}]}\right)$.

It is also worth considering that random deviations from perfect bilateral symmetry (fluctuating asymmetry), which are thought to indicate developmental instability, vary across the menstrual cycle [25]. Furthermore, associations between 2D:4D and facial asymmetry in males and females have been observed [26] (though see van Dongen [27]), and women with more symmetrical fourth finger lengths have been shown to have higher levels of oestradiol [28]. Although $\mathrm{D}_{[\mathrm{R}-\mathrm{L}]}$ relates to directional asymmetry (i.e. the values are signed) not fluctuating 
asymmetry (for which the values are unsigned), it is possible that the observations made by the current study reflect circulating hormonal influences on bodily asymmetry rather than the organisational effects of prenatal sex hormones.

Although trend level positive correlations were observed at the peri-ovulatory and luteal phases, the current study observed no significant correlations between digit ratio and progesterone levels across the menstrual cycle, which is consistent with the findings of previous studies $[15,16]$. Further to this, no significant associations were observed between digit ratio and testosterone. Although no study had previously examined this possible association across the menstrual cycle, the null-finding is consistent with previous research showing no association between digit ratio and circulating testosterone levels in adult females [11]. It is nevertheless interesting to note that Ventura et al. [29] more recently reported negative correlations between circulating testosterone measured from pregnant females during the second trimester, and both R2D:4D and L2D:4D. These effects were however observed only when the developing foetus was male, and the findings from this study are unlikely to be generalisable to naturally cycling females.

Although most research on 2D:4D has focussed on its potential relationship with foetal testosterone [30], it has been suggested that foetal oestrogen should not be overlooked, and indeed that it may be the balance between these hormones that is of greatest importance $[3,4]$. This idea is supported by Lutchmaya et al. [31], who found that T:O ratio in amniotic fluid predicted subsequent R2D:4D in two-year-old infants. Muller et al. [9] examined circulating $\mathrm{T}: \mathrm{O}$, and reported a significant association with R2D:4D in a large sample $(n=1036)$ of adult males. Although Muller et al. [9] observed no significant effect in females $(n=620)$, this could be because the sample was comprised only of postmenopausal women. Considering the marked differences in hormonal levels and lack of ovarian synthesis of hormones in post-menopausal women, it may be that associations between digit ratio and $\mathrm{T}: \mathrm{O}$ ratio in females are only observable during pre-menopause. The current study observed a marginally significant negative correlation between $\mathrm{D}_{[\mathrm{R}-\mathrm{L}]}$ and follicular T:O. This suggests that the effect may be worth examining further in larger samples of naturally cycling females.

A strength of the current study is that we determined ovulation (in 22 of 32 women studied) from LH tests, which may be more accurate than methods used in earlier studies (McIntyre et al. [15] did not independently assess ovulation; Klimek et al. [16] determined ovulation by 
mid-cycle oestradiol drop day). However, some limitations must also be acknowledged. We did not measure 2D:4D directly, but instead used photocopied images, which are known to yield lower (i.e. more masculinised) ratios [32,33]. However, it should also be noted that computer-assisted analysis of digit ratios produces more accurate and consistent measurements among observers [34], and several previous studies of 2D:4D in relation to the menstrual cycle have used photocopies or scans [15,20,35]. It is also worth pointing out that Mayhew et al. [35] reported digit ratios to differ significantly across different phases of the menstrual cycle. However, two subsequent studies $[20,36]$ showed stable values of digit ratios across the cycle.

Another limitation of the current study is that the sample size was small. However, it should be noted that it was not dissimilar to those of some previous studies in the area [11]. We therefore suggest that the effects not previously examined (e.g. the possible association between digit ratio and $\mathrm{T}: \mathrm{O}$ ratio in females of reproductive age) should be further investigated in larger samples.

\section{Conclusions}

The current study provides evidence consistent with previous research to suggest that digit ratio is not strongly associated with adult circulating progesterone or testosterone in naturally cycling females. The study also observed that $\mathrm{D}_{[\mathrm{R}-\mathrm{L}]}$ was positively associated with oestradiol levels measured throughout the menstrual cycle, a finding that is consistent with that of McIntyre et al. [15]. However, these findings should be interpreted with caution considering that both McIntyre et al. [15] and the current study reported on small samples, whereas a larger study [16] observed no significant correlations between digit ratio and circulating hormone levels across the menstrual cycle.

\section{Conflict of interest statement}

None declared.

\section{Role of the funding source}

This work was supported by the Polish National Science Centre [grant number 2014/12/S/NZ8/00722 to UMM]. The funder played no role in study design, collection, analysis and interpretation of data, in the writing of the manuscript, or in the decision to submit 
the article for publication.

\section{Author contributions}

GR analysed and interpreted the data, and wrote the manuscript. UMM and MK recruited participants and collected the data. MK, GJ, and UMM designed the study, and revised the manuscript for important intellectual content. All authors read and approved the final version of the manuscript.

\section{References}

[1] J.T. Manning, D. Scutt, J. Wilson, D.I. Lewis-Jones, The ratio of 2nd to 4th digit length: a predictor of sperm numbers and levels of testosterone, luteinizing hormone and oestrogen, Hum. Reprod. 13 (1998) 3000-3004, http://dx.doi.org/10.1093/humrep/13.11.3000.

[2] J.T. Manning, Digit Ratio: a Pointer to Fertility, Behaviour and Health, Rutgers University Press, New Brunswick, NJ, 2002.

[3] Z. Zheng, M.J. Cohn, Developmental basis of sexually dimorphic digit ratios, Proc. Natl. Acad. Sci. 108 (2011) 16289-16294, http://dx.doi.org/10.1073/pnas.1108312108.

[4] J.T. Manning, Resolving the role of prenatal sex steroids in the development of digit ratio, Proc. Natl. Acad. Sci. 108 (2011) 16143-16144, http://dx.doi.org/10.1073/pnas.1113312108.

[5] J. Hönekopp, S. Watson, Meta-analysis of digit ratio 2D:4D shows greater sex difference in the right hand, Am. J. Hum. Biol. 22 (2010) 619-630, http://dx.doi.org/10.1002/ajhb.21054.

[6] S.A. Berenbaum, K.K. Bryk, N. Nowak, C.A. Quigley, S. Moffat, Fingers as a marker of prenatal androgen exposure, Endocrinology 150 (2009) 5119-5124, http://dx.doi.org/10.1210/en.20090774 .

[7] J. van Hemmen, P.T. Cohen-Kettenis, T.D. Steensma, D.J. Veltman, J. Bakker, Do sex differences in CEOAEs and 2D:4D ratios reflect androgen exposure? A study in women with complete androgen insensitivity syndrome, Biol. Sex Differ. 8 (2017) 11, http://dx.doi.org/10.1186/s13293-017-0132-z.

[8] J.T. Manning, S. Wood, E. Vang, J. Walton, P.E. Bundred, C. van Heyningen, D.I. Lewis-Jones, Second to fourth digit ratio (2D:4D) and testosterone in men, Asian J. Androl. 6 (2004) 211215. 
[9] D.C. Muller, G.G. Giles, J. Bassett, H.A. Morris, J.T. Manning, J.L. Hopper, D.R. English, G. Severi, Second to fourth digit ratio (2D:4D) and concentrations of circulating sex hormones in adulthood, Reprod. Biol. Endocrinol. 9 (2011) 57, http://dx.doi.org/10.1186/1477-7827-9-57.

[10] M. Klimek, A. Galbarczyk, I. Nenko, L.C. Alvarado, G. Jasienska, Digit ratio (2D:4D) as an indicator of body size, testosterone concentration and number of children in human males, Ann. Hum. Biol. 41 (2014) 518-523, http://dx.doi.org/10.3109/03014460.2014.902993.

[11] J. Hönekopp, L. Bartholdt, L. Beier, A. Liebert, Second to fourth digit length ratio (2D:4D) and adult sex hormone levels: new data and a meta-analytic review, Psychoneuroendocrinology 32 (2007) 313-321, http://dx.doi.org/10.1016/j.psyneuen.2007.01.007.

[12] L. Kilduff, C.J. Cook, M. Bennett, B. Crewther, R.M. Bracken, J.T. Manning, Right-left digit ratio (2D:4D) predicts free testosterone levels associated with a physical challenge, J. Sports Sci. 31 (2013) 677-683, http://dx.doi.org/10.1080/02640414.2012.747690.

[13] B. Crewther, C.J. Cook, L. Kilduff, J.T. Manning, Digit ratio (2D:4D) and salivary testosterone, oestradiol and cortisol levels under challenge: evidence for prenatal effects on adult endocrine responses, Early Hum. Dev. $91 \quad$ (2015) 451-456, http://dx.doi.org/10.1016/j.earlhumdev.2015.04.011.

[14] J.T. Manning, L. Kilduff, C. Cook, B. Crewther, B. Fink, Digit ratio (2D:4D): a biomarker for prenatal sex steroids and adult sex steroids in challenge situations, Front. Endocrinol. 5 (2014), http://dx.doi.org/10.3389/fendo.2014.00009.

[15] M.H. McIntyre, J. Flynn Chapman, S.F. Lipson, P.T. Ellison, Index-to-ring finger ratio (2D:4D) predicts levels of salivary estradiol, but not progesterone, over the menstrual cycle, Am. J. Hum. Biol. 19 (2007) 434-436, http://dx.doi.org/10.1002/ajhb.20623.

[16] M. Klimek, A. Galbarczyk, H. Colleran, I. Thune, P.T. Ellison, A. Ziomkiewicz, G. Jasienska, Digit ratio (2D:4D) does not correlate with daily $17 \beta$-estradiol and progesterone concentrations in healthy women of reproductive age, Am. J. Hum. Biol. 27 (2015) 667-673, http://dx.doi.org/10.1002/ajhb.22717.

[17] G. Jasienska, The Fragile Wisdom. An Evolutionary View on Women's Biology and Health, Harvard University Press, Cambridge, MA, 2013.

[18] E. Guermandi, W. Vegetti, M.M. Bianchi, A. Uglietti, G. Ragni, P. Crosignani, Reliability of ovulation tests in infertile women, Obstet. Gynecol. 97 (2001) 92-96, http://dx.doi.org/10.1016/s0029-7844(00)01083-8.

[19] O.C. Schultheiss, S.J. Stanton, Assessment of salivary hormones, in: E. Harmon-Jones, J.S. Beer (Eds.), Methods in Social Neuroscience, Guilford Press, New York, NY, 2009, pp. 17-44. 
[20] M. Klimek, U.M. Marcinkowska, G. Jasienska, Value of digit ratio 2D:4D, a biomarker of prenatal hormone exposure, is stable across the menstrual cycle, Early Hum. Dev. 110 (2017) 21-24, http://dx.doi.org/10.1016/j.earlhumdev.2017.04.014.

[21] Y. Kaneoke, T. Donishi, A. Iwahara, T. Shimokawa, Severity of premenstrual symptoms predicted by second to fourth digit ratio, Front. Med. 4 (2017) 144, http://dx.doi.org/10.3389/fmed.2017.00144.

[22] U. Halbreich, The etiology, biology, and evolving pathology of premenstrual syndromes, Psychoneuroendocrinology $28 \quad$ (2003) 55-99, http://dx.doi.org/10.1016/S03064530(03)00097-0.

[23] J.M. Tanner, Foetus into Man: Physical Growth from Conception to Maturity, Harvard University Press, Cambridge, MA, 1990.

[24] D. Kimura, Body asymmetry and intellectual pattern, Personal. Individ. Differ. 17 (1994) 53-60, http://dx.doi.org/10.1016/0191-8869(94)90261-5.

[25] J.T. Manning, D. Scutt, G.H. Whitehouse, S.J. Leinster, J.M. Walton, Asymmetry and the menstrual cycle in women, Ethol. Sociobiol. 17 (1996) 129-143, http://dx.doi.org/10.1016/0162-3095(96)00001-5.

[26] B. Fink, J.T. Manning, N. Neave, K. Grammer, Second to fourth digit ratio and facial asymmetry, Evol. Hum. Behav. 25 (2004) 125-132, http://dx.doi.org/10.1016/S1090-5138(03)00084-9.

[27] S. van Dongen, A critical re-evaluation of the association between 2D:4D ratios and fluctuating asymmetry in humans, Ann. Hum. Biol. 36 (2009) 186-198, http://dx.doi.org/10.1080/03014460802691182.

[28] G. Jasienska, S.F. Lipson, P.T. Ellison, I. Thune, A. Ziomkiewicz, Symmetrical women have higher potential fertility, Evol. Hum. Behav. 27 (2006) 390-400, http://dx.doi.org/10.1016/j.evolhumbehav.2006.01.001.

[29] T. Ventura, M.C. Gomes, A. Pita, M.T. Neto, A. Taylor, Digit ratio (2D:4D) in newborns: influences of prenatal testosterone and maternal environment, Early Hum. Dev. 89 (2013) 107112, http://dx.doi.org/10.1016/j.earlhumdev.2012.08.009.

[30] S.M. Breedlove, Minireview. Organizational hypothesis: instances of the fingerpost, Endocrinology 151 (2010) 4116-4122, http://dx.doi.org/10.1210/en.2010-0041.

[31] S. Lutchmaya, S. Baron-Cohen, P. Raggatt, R. Knickmeyer, J.T. Manning, 2nd to 4th digit ratios, fetal testosterone and estradiol, Early Hum. Dev. 77 (2004) 23-28, http://dx.doi.org/10.1016/j.earlhumdev.2003.12.002. 
[32] J.T. Manning, B. Fink, N. Neave, N. Caswell, Photocopies yield lower digit ratios (2D:4D) than direct finger measurements, Arch. Sex. Behav. 34 (2005) 329-333, http://dx.doi.org/10.1007/s10508-005-3121-y.

[33] E. Ribeiro, N. Neave, R.N. Morais, J.T. Manning, Direct versus indirect measurement of digit ratio (2D:4D): a critical review of the literature and new data, Evol. Psychol. 14 (2016) 1-8, http://dx.doi.org/10.1177/1474704916632536.

[34] H.C. Allaway, T.G. Bloski, R.A. Pierson, M.E. Lujan, Digit ratios (2D:4D) determined by computer-assisted analysis are more reliable than those using physical measurements, photocopies, and printed scans, Am. J. Hum. Biol. 21 (2009) 365-370, http://dx.doi.org/10.1002/ajhb.20892.

[35] T.M. Mayhew, L. Gillam, R. McDonald, F.J. Ebling, Human 2D (index) and 4D (ring) digit lengths: their variation and relationships during the menstrual cycle, J. Anat. 211 (2007) 630638, http://dx.doi.org/10.1111/j.1469-7580.2007.00801.x.

[36] E.S. Barrett, L.E. Parlett, S.H. Swan, Stability of proposed biomarkers of prenatal androgen exposure over the menstrual cycle, J. Dev. Orig. Health Dis. 6 (2015) 149-157, http://dx.doi.org/10.1017/s2040174414000646. 